\title{
A Novel Approach on Parameter Identification for Inverter Driven Induction Machines
}

\author{
Richard F. F. Koning, Chun Tung Chou, Michel H. G. Verhaegen, J. Ben Klaassens, and Jeroen R. Uittenbogaart
}

\begin{abstract}
AC machines are applied in actuator systems for many applications. The development of fast processors open up the possibilities to apply new techniques for identification and control of electrical drives in real-time, so as to improve and optimize their performances. The paper presents a systematic approach to identify the physically relevant parameters of an ac machine. Special attention is paid to the identification of these parameters, without differentiating the measured voltages and currents. This approach allows the use of high sampling rates necessary to apply identification in real time. The approach is applicable for electrical machines but can be extended to other classes of electro-mechanical actuators. The simulation studies performed in this paper highlight the improvements of the new outlined approach over existing identification methods under realistic operation conditions where the test signals are generated by the power source (inverter) in an uncomplicated way.
\end{abstract}

Index Terms-AC machines, continuous-time parameter identification, inverters, linear regression, noise, nonlinear systems.

\section{NOMENCLATURE}

$u_{s \alpha}, u_{s \beta} \quad \alpha$ - and $\beta$-phase of the stator voltage in the stationary reference frame.

$i_{s \alpha}, i_{s \beta} \quad \alpha$ - and $\beta$-phase of the stator current in the stationary reference frame.

$i_{R \alpha}, i_{s \beta} \quad \alpha$ - and $\beta$-phase of the rotor current in the stationary reference frame.

$\psi_{R \alpha}, \psi_{R \beta} \quad \alpha$ - and $\beta$-phase of the rotor flux in the stationary reference frame.

$\omega_{r} \quad$ Electrical rotor speed $[\mathrm{rad} / \mathrm{s}]$.

$\omega_{s} \quad$ Stator frequency $[\mathrm{rad} / \mathrm{s}]$.

$R_{s} \quad$ Stator resistance.

$R_{R} \quad$ Rotor resistance in $\Gamma^{\prime}$-scheme.

$L_{l} \quad$ Leakage inductance in $\Gamma^{\prime}$-scheme.

Manuscript received June 17, 1996; revised November 5, 1997. Recommended by Associate Editor, J.M. Maciejowski. Thwork of J. R. Uittenbogaart was done while he was with the Institute of Applied Physics of the Netherlands Organization for Applied Scientific Research.

R. F. F. Koning, C. T. Chou, and J. B. Klaassens are with the Faculty of Information Technology and Systems, Department of Electrical Engineering, Systems and Control Laboratory, Delft University of Technology, Delft 2628 CD, The Netherlands (e-mail: j.b.klaassens@its.tudelft.nl).

M. H. G. Verhaegen was with the Faculty of Information Technology and Systems, Department of Electrical Engineering, Systems and Control Laboratory, Delft University of Technology, Delft NL-2600 GA, The Netherlands. He is now with the Faculty of Applied Physics, University of Twente, Enschede 7500 AE, The Netherlands (e-mail: m.verhaegen@tn.utwente.nl).

J. R. Uittenbogaart is with the TNO Institute of Applied Physics, NL-2600 AD Delft, The Netherlands.

Publisher Item Identifier S 1063-6536(00)05753-5.
$\begin{array}{ll}L_{m} & \text { Mutual inductance in } \Gamma^{\prime} \text {-scheme } \\ s & \text { Slip }=\left(\omega_{s}-\omega_{r}\right) / \omega_{s} .\end{array}$

\section{INTRODUCTION}

$\mathbf{T}$ HE INDUCTION motor and power electronic converters are now an important combination for high-performance adjustable speed drives and servodrives. To achieve good performance, the motor parameters have to be known accurately under all regular and irregular conditions of operation. Parameter mismatch is the most dominant remaining problem in field-oriented control. There are several reasons for a mismatch like temperature and magnetic saturation.

Measurements of stator voltages and currents, sometimes together with rotor speed measurement, are used in a number of identification approaches to determine stator and rotor impedances in a linear state-space model with time varying parameters representing the induction machine dynamics [5], [22], [26]. In spite of the fact that a number of nonlinear parasitic effects like magnetic saturation, hysteresis, and eddy currents are neglected, the existing solutions are leading to inaccurate and/or very large calculations. Real-time model-based controls are therefore not realizable [26].

The no-load and locked rotor tests have been the methods in practice to estimate the parameters of an induction motor, using a steady-state equivalent-circuit model. It is a simple method but two different test configurations are required to determine all parameters. This off-line method needs special test signals.

This equivalent-circuit model is also used in more recently developed methods to estimate the parameters of an induction motor. The standstill frequency response method (SSFR) uses frequency domain data to estimate the parameter values [9]. A single test configuration is sufficient to determine the parameters. It is, however, an off-line method using special test signals in a special test configuration.

In other methods special test signals are injected into the machine and the parameters are directly calculated from the time-response data [3], [6], [10]. Such methods are also used in self-commissioning schemes that have been developed [7], [17]. In [24] the maximum-likelihood (ML) method is used to estimate the equivalent-circuit model parameters from a standstill time-domain response.

All the methods using the equivalent-circuit model to estimate the motor parameters have the disadvantage of being off-line methods due to the special test signals and configurations. 
Parameter estimation methods that use the dynamical equations of an induction motor have been proposed. These methods can be divided into analytical methods, statistical methods and methods using an extended Kalman filter (EKF). In the analytical methods algebraic equations are derived from the nonlinear differential equations by which the parameters can be calculated out of voltage and current measurements [3], [11].

In statistical approaches least squared error models are usually used for parameter fitting, using regression techniques. These methods are however based on linear equation models. To apply these methods, a number of different approaches have been presented to derive linearly parameterized induction motor models from the nonlinear model equations [12], [25], [27]. In [18] the steepest gradient method is used to fit the measured data directly to the nonlinear equation model. This method can be used on-line because special test signals are not required. However, the performance depends on the richness of the excitation that cannot always be guaranteed under normal conditions of operation.

There are a number of adaptive approaches that estimate the rotor resistance or rotor time constant by injecting special excitation signals into the motor [2], [4], [13], [29] but most adaptive approaches use a so-called model reference adaptive control (MRAC) structure. The different MRAC methods that have been reported differ mostly in the used reference model. This includes for example reference models for the reactive power [1], for the electromagnetic torque [8] and for the rotor flux [14], [20].

Another recently developed method uses an EKF for on-line estimation of the rotor resistance [19].

These methods enable on-line estimation of parameters directly used in field-oriented controllers. Most adaptive methods only deal with varying rotor parameters. A major drawback of the existing EKF and other nonlinear parameter optimization procedures is the need of suitable initial estimates.

The contribution of this paper is the presentation of a new technique to identify linear dynamic models by a method of linear regression without differentiation. The method allows online estimation of parameters of induction machines powered by switching waveforms and provides initial estimates to EKF type of procedures.

The model of the induction machine and a formulation of the Parameter estimation problem is presented in Section II. An outline of the solution is given in Section III where the basic aspects of the approach developed in [28] are briefly

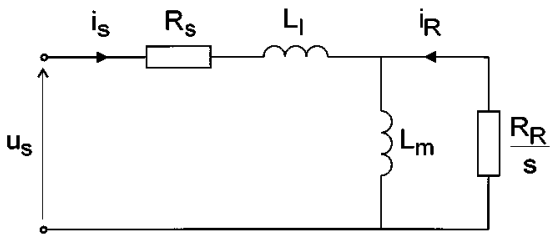

Fig. 1. Steady-state representation of the induction motor.

outlined. Results are given in Section IV. Section V presents the conclusions.

\section{Model of AN INDUCTION Motor AND Estimating THE MOTOR PARAMETERS}

The induction motor is represented by its state-space model in a fixed reference frame, which is also known as the $\Gamma^{\prime}$-scheme [21]

$$
\begin{aligned}
\frac{d}{d t}\left[\begin{array}{c}
i_{s \alpha} \\
i_{s, \beta} \\
\Psi_{R \alpha} \\
\Psi_{R \beta}
\end{array}\right] & {\left[\begin{array}{ccrc}
-\frac{R_{s}+R_{R}}{L_{l}} & 0 & \frac{R_{R}}{L_{m} L_{l}} & \frac{\omega_{r}}{L_{l}} \\
0 & -\frac{R_{s}+R_{R}}{L_{l}} & -\frac{\omega_{r}}{L_{l}} & \frac{R_{R}}{L_{m} L_{l}} \\
R_{R} & 0 & -\frac{R_{R}}{L_{m}} & -\omega_{r} \\
0 & 0 & \omega_{r} & -\frac{R_{R}}{L_{m}}
\end{array}\right] } \\
& \cdot\left[\begin{array}{c}
i_{s \alpha} \\
i_{s, \beta} \\
\Psi_{R \alpha} \\
\Psi_{R \beta}
\end{array}\right]+\left[\begin{array}{cc}
\frac{1}{L_{l}} & 0 \\
0 & \frac{1}{L_{l}} \\
0 & 0 \\
0 & 0
\end{array}\right]\left[\begin{array}{l}
u_{s \alpha} \\
u_{s, \beta}
\end{array}\right] .
\end{aligned}
$$

The rotor speed $\omega_{r}$ is the mechanical speed of the motorshaft $\omega_{r}=(1-s) \omega_{s}$ where $s$ is the slip and $\omega_{s}$ the stator frequency. If we denote the differential-operator as " $D$ " then the motor equations can be rewritten by eliminating the rotorflux in equation (1) as shown in (2) at the bottom of the page. The steady-state representation of this model is given in Fig. 1.

$L_{m}=(1-\sigma) L_{s}$ and $L_{l}=\sigma L_{s}$ where $\sigma$ is the coefficient of coupling.

$$
\left[\begin{array}{c}
u_{s \alpha} \\
u_{s \beta} \\
0 \\
0
\end{array}\right]=\left[\begin{array}{cccc}
R_{s}+D\left(L_{l}+L_{m}\right) & 0 & D L_{m} & 0 \\
0 & R_{s}+D\left(L_{l}+L_{m}\right) & 0 & D L_{m} \\
D L_{m} & \omega_{r} L_{m} & R_{R}+D L_{m} & \omega_{r} L_{m} \\
-\omega_{r} L_{m} & D L_{m} & -\omega_{r} L_{m} & R_{R}+D L_{m}
\end{array}\right]\left[\begin{array}{c}
i_{s \alpha} \\
i_{s \beta} \\
i_{R \alpha} \\
i_{R \beta}
\end{array}\right]
$$


The currents $i_{R \alpha}$, and $i_{R \beta}$ and their derivatives are not measurable quantities. If we eliminate the unmeasurable quantities from equation (2), two differential equations result

$$
\begin{aligned}
& a_{2} \frac{d^{2} u_{s \alpha}}{d t^{2}}+a_{1} \frac{d u_{s \alpha}}{d t}+a_{0} u_{s \alpha} \\
& \quad=-b_{1} \frac{d i_{s \beta}}{d t}+c_{3} \frac{d^{3} i_{s \alpha}}{d t^{3}}+c_{2} \frac{d^{2} i_{s \alpha}}{d t^{2}}+c_{1} \frac{d i_{s \alpha}}{d t}+c_{0} i_{s \alpha} \\
& a_{2} \frac{d^{2} u_{s, \beta}}{d t^{2}}+a_{1} \frac{d u_{s \beta}}{d t}+a_{0} u_{s \beta} \\
& \quad=b_{1} \frac{d i_{s \alpha}}{d t}+c_{3} \frac{d^{3} i_{s \beta}}{d t^{3}}+c_{2} \frac{d^{2} i_{s \beta}}{d t^{2}}+c_{1} \frac{d i_{s \beta}}{d t}+c_{0} i_{s \beta} .
\end{aligned}
$$

Because the coefficients of (3) and (4) are the same, we can identify these coefficients using only one of these equations.

In the following (3) will be used. It is sufficient to measure $i_{s \alpha}, i_{s \beta}$ and $u_{s \alpha}$, The coefficients of the differential equations (3) and (4) are related to the physical parameters in (1) by the following relations:

$$
\begin{aligned}
& a_{2}=L_{m}^{2} \quad a_{1}=2 L_{m} R_{R} \quad a_{0}=\omega_{r}^{2} L_{m}^{2}+R_{R}^{2} \\
& b_{1}=\omega_{r} L_{m}^{2} R_{R} \quad c_{3}=L_{m}^{2} L_{l} \\
& c_{2}=L_{m}^{2} R_{R}+L_{m}^{2} R_{s}+2 R_{R} L_{l} L_{m} \\
& c_{1}=\omega_{r}^{2} L_{m}^{2} L_{l}+L_{m} R_{R}^{2}+2 R_{R} R_{s} L_{m}+L_{l} R_{R}^{2} \\
& c_{0}=R_{s} R_{R}^{2}+\omega_{r}^{2} L_{m}^{2} R_{s} .
\end{aligned}
$$

Define $\Theta=\left[a_{2}, \ldots, c_{0}\right]^{T}$ and $x=\left(L_{m}, L_{l}, R_{s}, R_{R}\right)$, then we can denote this set (5) as

$$
\Theta=F(x) .
$$

Now we can define the following parameter estimation problem.

Consider an induction motor presented by its continuous-time state-space model (1) with unknown parameters $L_{l}, L_{m}, R_{s}, R_{R}$. Available are the sampled data sequences of the stator currents $i_{s \alpha, m}, i_{s \beta, m}$, voltages $u_{s \alpha}$ and rotor speed $\omega_{r}$. The task is to determine the motor parameters $L_{l}, L_{m}, R_{s}$ and $R_{R}$.

This parameter estimation problem is formulated mathematically as

$$
\min _{x} \sum_{t=1}^{N}\left(i_{s \alpha, m}(t)-i_{s \alpha}(t, x)\right)^{2}
$$

where $i_{s \alpha}(t, x)$ is the solution to the differential equation (3) with coefficients $a_{i}, b_{i}$ and $c_{i}$ determined by $x=\left(L_{m}, L_{l}, R_{s}, R_{R}\right)$. From this general problem statement two special cases are considered: constant rotor speed and varying rotor speed.

1) Constant Rotor Speed: When $\omega_{r}$ is constant the coefficients of equation (3) are constant and this equation can be written as a linear time-invariant transfer function

$$
\begin{aligned}
i_{s \alpha}(s)= & \frac{b_{1} s}{c_{3} s^{3}+c_{2} s^{2}+c_{1} s+c_{0}} i_{s \beta}(s) \\
& +\frac{a_{2} s^{2}+a_{1} s+a_{0}}{c_{3} s^{3}+c_{2} s^{2}+c_{1} s+c_{0}} u_{s \alpha}(s)
\end{aligned}
$$

where $s$ is the Laplace operator.
The coefficients of this transfer function are identified using the method developed in [28] and is summarized in the next section.

2) Varying Rotor Speed: If the rotor speed $\omega_{r}$ is a timevarying function and the rotor speed can be measured, the equations can be transformed so that the new coefficients become constant again. To apply the identification techniques presented in [28], a relationship between measurable quantities in the Laplace domain is necessary. The Laplace transform of a product of two time-varying functions results in a convolution in the Laplace domain. For example

$$
\mathcal{L}\left(\omega_{r} \frac{d i_{s \alpha}}{d t}\right)=\omega_{r}(s) * s i_{s \alpha}(s)
$$

To avoid this, the following substitution using the chain rule is made

$$
\omega_{r} \frac{d i_{s \alpha}}{d t}=\frac{d\left(\omega_{r} i_{s \alpha}\right)}{d t}-i_{s \alpha} \frac{d \omega_{r}}{d t} .
$$

While the rotor speed $\omega_{r}$ changes relatively slow and is a smooth function of time, its derivative is accurately calculated by making use of, e.g., the spline interpolation. This will yield more accurate results compared to differentiating the high-frequency currents and voltages, because in practice these signals are not smooth or contain spikes. Based on the idea outlined by (10), we can get a set of equations in terms of signals that can be derived from the measured data

$$
\begin{aligned}
d_{2} \frac{d^{2} u_{s \alpha}}{d t^{2}}+d_{1} \frac{d u_{s \alpha}}{d t}+d_{0} u_{s \alpha}+e_{0}\left(\omega_{r}^{2} u_{s \alpha}\right) \\
\quad+f_{1}\left(\frac{d\left(\omega_{r} i_{s \beta}\right)}{d t}-i_{s \beta} \frac{d \omega_{r}}{d t}\right) \\
=g_{3} \frac{d^{3} i_{s \alpha}}{d t^{3}}+g_{2} \frac{d^{2} i_{s \alpha}}{d t^{2}}+g_{1} \frac{d i_{s \alpha}}{d t}+g_{0} i_{s \alpha} \\
\quad+h_{1}\left(\frac{d\left(i_{s \alpha} \omega_{r}^{2}\right)}{d t}-2 i_{s \alpha} \omega_{r} \frac{d \omega_{r}}{d t}\right)+k_{0}\left(\omega_{r}^{2} i_{s \alpha}\right) .
\end{aligned}
$$

These coefficients are related to the parameters of the $\Gamma^{\prime}$-scheme

$$
\begin{aligned}
& d_{2}=L_{m}^{2} \quad d_{1}=2 L_{m} R_{R} \quad d_{0}=R_{R}^{2} \\
& e_{0}=L_{m}^{2} \quad f_{1}=L_{m}^{2} R_{R} \quad g_{3}=L_{m}^{2} L_{l} \\
& g_{2}=L_{m}^{2} R_{R}+L_{m}^{2} R_{s}+2 R_{R} L_{l} L_{m} \\
& g_{1}=L_{m} R_{R}^{2}+2 R_{R} R_{s} L_{m}+L_{l} R_{R}^{2} \\
& g_{0}=R_{s} R_{R}^{2} \quad h_{1}=L_{m}^{2} L_{l} \quad k_{0}=L_{m}^{2} R_{s} .
\end{aligned}
$$

The identification problem can now be formulated in the same way as for the constant rotor speed case. Therefore, without loss of generality, we restrict this paper to outlining the improvements that may be obtained using the identification strategy highlighted in the Section III for the case $\omega_{r}$ is constant.

\section{OUtLine OF THE SOlution STRATEGY FOR CONSTANT $\omega_{r}$}

The identification problem (7) can be solved via nonlinear optimization techniques. To avoid the numerical difficulties (such as local minima) related to the use of such techniques, in this section a different strategy is outlined. This strategy separates the identification problem into subproblems: a linear-in-the-pa- 
rameters optimization problem and finding the zeros of a simple nonlinear function.

Mathematically, these steps are formulated as

$$
\text { step 1: } \min _{\Theta} \sum_{t=1}^{N}\left(I_{s \alpha, m}(t)-i_{s \alpha}(t, \Theta)\right)^{2}
$$

where $\Theta$ is defined in (6). Denote the solution of this step by $\hat{\Theta}$

$$
\text { step 2: } \quad \min _{x}\|F(x)-\hat{\Theta}\|_{2}^{2} \text {. }
$$

Here the parameter vectors $\Theta, x$ and the vector function $F(x)$ are as given by (5) and (6). Based on physical insight the optimization problem in (14) can be constrained by including the condition $x>O$.

In the first step, the coefficients of the transfer function are found from the measured input and output data.

In the second step the motor parameters are estimated from the transfer function coefficients. There is some redundancy in the number of equations in step 2: there are eight equations but only four unknown parameters. Later this will be exploited by solving this step using only the most accurate estimates that still allow a unique solution of $L_{l}, L_{m}, R_{s}$, and $R_{R}$. Therefore, the key problem is to find the coefficients of a differential equation.

The problem of modeling a continuous-time process from discrete-time data can be solved in different ways.

One possible way is known as the indirect method. It estimates the continuous-time parameters by first fitting a discrete-time model to the data and then converting this model to a continuous-time model.

Another approach is to approximate the continuous-time derivatives by finite differences [23] or, alternatively, integrals by sums. This approach results in the possibility of estimating the continuous-time system from the discrete-time data without an intermediate step. This is called a direct method. The calculation of derivatives however can lead to serious problems if the data is corrupted with noise or has steep waveforms as we will see in Section IV.

To avoid the calculation of derivatives, we will use an identification method using all-pass filters [28]. A summary of this approach is outlined next.

\section{A. Estimating the Parameter in a Differential Equation}

The solution described in [28] performs a bilinear transformation on the differential operator in the continuous differential equation. As a consequence the measured inputs and output are filtered through a bank of all-pass filters. It is shown that the filtered inputs, filtered output and the impulse response of the filters are related by a linear algebraic equation. Therefore, the coefficients in this algebraic equation can be determined using linear least-square type of techniques. It is shown in [28] that there exists an analytic, linear transformation between the coefficients of the differential equation and those of the difference equation. The desired coefficients ofthe differential equation can readily be recovered once the coefficients of the alge- braic equation are computed. This procedure is outlined for the special case of identifying a first-order continuous time system.

Let the bilinear transformation of the Laplace operator, denoted by $w$, be defined as

$$
w=\frac{s+\lambda}{s-\lambda}
$$

where $\lambda<O$

The inverse relationship is

$$
s=\lambda \frac{1+w}{1-w} .
$$

Given the following first-order system:

$$
\left(a_{1} s+a_{0}\right) Y(s)=\left(b_{1} s+b_{0}\right) U(s)+c_{0} \quad\left(a_{1} \neq 0\right)
$$

where $U(s)$ and $Y(s)$ are the Laplace transforms of the input $u(t)$ and output $y(t)$, respectively. If the system response due to the unknown initial condition is given by $c_{0}$ then the equation in terms of the $w$-operator becomes

$$
\begin{aligned}
& {\left[\left(a_{1} \lambda-a_{0}\right) w+\left(a_{1} \lambda+a_{0}\right)\right] Y(s)} \\
& \quad=\left[\left(b_{1} \lambda-b_{0}\right) w+\left(b_{1} \lambda+b_{0}\right)\right] U(s)+\left(-c_{0} w+c_{0}\right)
\end{aligned}
$$

denoted more compactly as

$$
\left(\bar{a}_{1} w+\bar{a}_{0}\right) Y(s)=\left(\bar{b}_{1} w+\bar{b}_{0}\right) U(s)+\left(\bar{c}_{1} w+\bar{c}_{0}\right) .
$$

Clearly there is a linear relation between the coefficients in (17) and (19). In terms of time domain signals the equation (19) becomes

$$
\bar{a}_{1} \bar{y}(t)+\bar{a}_{0} y(t)=\bar{b}_{1} \bar{u}(t)+\bar{b}_{0} u(t)+\bar{c}_{1} \bar{w}(t)+\bar{c}_{0} \delta(t)
$$

where

$$
\bar{y}=[w y](t) \quad \bar{u}=[w u](t) \quad \bar{w}=[w \delta](t)
$$

$\delta(t)$ is the continuous time impulse function and $\bar{y}$ denotes the inverse Laplace transformation of $w(s) Y(s)$.

Now, define the matrices: $\bar{Y}, \bar{U}$, and $\bar{W}$ as

$$
\begin{aligned}
& \bar{Y}=\left[\begin{array}{cc}
\bar{y}(0) & y(0) \\
\vdots & \vdots \\
\bar{y}(N-1) & y(N-1)
\end{array}\right] \\
& \bar{U}=\left[\begin{array}{cc}
\bar{u}(0) & u(0) \\
\vdots & \vdots \\
\bar{u}(N-1) & u(N-1)
\end{array}\right] \\
& \bar{W}=\left[\begin{array}{cc}
\bar{w}(0) & 1 \\
\vdots & \vdots \\
\bar{w}(N-1) & 0
\end{array}\right] .
\end{aligned}
$$


Then the coefficients $\bar{a}_{i}, \bar{b}_{i}$, and $\bar{c}_{i}$ satisfy

$$
[\bar{W}|\bar{Y}| \bar{U}]\left[\begin{array}{c}
\bar{c}_{1} \\
\bar{c}_{0} \\
\overline{-\bar{a}_{1}} \\
-\bar{a}_{0} \\
\overline{\bar{b}_{1}} \\
\bar{b}_{0}
\end{array}\right]=0 .
$$

This shows that up to a proportional constant they form the solution of a so-called total least squares (TLS) problem [15].

An important step in the above procedure is the all-pass filtering of the input-output data. Since we only have sampled data sequences we have to use a digital approximation of the continuous time filter (15). Different higher-order approximation schemes are discussed in [28]. For simplicity in this paper we restrict to the first-order Padé approximation or Tustin transformation. Let $T_{s}$ be the sampling period, then discretization of (15) reads

$$
w_{d}(z)=\frac{\mathcal{X} z-1}{z-\mathcal{X}} \quad \text { where } \mathcal{X}=\frac{2-\lambda T_{s}}{2+\lambda T_{s}} .
$$

If this approximation scheme is selected then, under the conditions that the excitation is persistently exciting and that the error in the approximation of the continuous-time filter $w$ is negligible, the coefficients $\bar{a}_{i}, \bar{b}_{i}$ and $\bar{c}_{i}$ can be computed by solving the following TLS problem:

$$
\min _{\xi}\left\|\left[W \bar{Y}_{d} \bar{U}_{d}\right] \xi\right\|_{2} \quad \text { subject to } \xi^{T} \xi=1
$$

where $\|\ldots\|_{2}$ denotes the Euclidean length of the vector.

Finally, in order to force the differential equation to have a specific relative degree, additional constraints can be imposed on the TLS problem (25).

Since we are only interested in the coefficients $\bar{a}_{i}, \bar{b}_{i}$ and not in $\bar{c}_{i}$, we perform a QR-factorization on the matrix $[\bar{W}|\bar{Y}| \bar{U}]$, which compresses the amount of data and avoids calculating $\bar{c}_{i}$. Let this RQ factorization be denoted as

$$
[\bar{W} \mid \bar{Y} \bar{U}]=Q\left[\begin{array}{c|c}
R_{11} & R_{12} \\
0 & R_{22} \\
0 & 0
\end{array}\right]
$$

then $R_{22}$ gives the estimated values for the coefficients $\bar{a}_{i}$ and $\bar{b}_{i}$. Define $\bar{a}=\left[a_{n} \ldots a_{0}\right]^{T}$ and $\bar{b}=\left[b_{n} \ldots b_{0}\right]^{T}$ and $\bar{p}=\left[\begin{array}{l}\bar{a} \\ \bar{b}\end{array}\right]$ then an estimate of $\bar{p}$ denoted by $\hat{p}$ satisfies the following relationship:

$$
R_{22} \hat{p} \approx 0
$$

The solution to the equation above is not unique, because the modulus of the vector $\hat{p}$ is unknown. To obtain a unique solution we have to impose at least one constraint on $\hat{p}$, for example that $a_{i}=1$. For the induction motor, we also know that some coefficients are zero, because the degree of the numerator is lower than the degree of the denominator. The identification method allows the introduction of linear constraints on the coefficients $a_{i}$ and $b_{i}$. The constraints can be summarized in the matrix equation:

$$
C \bar{p}=d
$$

Now the following TLS problem has to be solved

$$
\min \|\Delta\|_{F}^{2} \text { such that }\left(R_{22}+\Delta\right) \hat{p}=0 \text { and } C \hat{p}=d \text {. }
$$

\section{B. Estimating the Motor Parameters}

The motor parameters are estimated from these coefficients once the coefficients of the transfer function are found. This problem can be stated as

$$
\min _{x}\|F(x)-\hat{\Theta}\|_{2}^{2}
$$

where $\hat{\Theta}$ contains the estimated transfer function coefficients.

The vector $x=\left[L_{m}, L_{l}, R_{s}, R_{R}\right]$ and $F(x)$ are given by

$$
\begin{aligned}
& F(x)=\left[\begin{array}{l}
a_{2} \\
a_{1} \\
a_{0} \\
b_{1} \\
c_{3} \\
c_{2} \\
c_{1} \\
c_{0}
\end{array}\right] \\
& =\left[\begin{array}{c}
L_{m}^{2} \\
2 L_{m} R_{R} \\
\omega_{r}^{2} L_{m}^{2}+R_{R}^{2} \\
\omega_{r} L_{m}^{2} R_{R} \\
L_{m}^{2} L_{l} \\
\omega_{r}^{2} L_{m}^{2} L_{l}+L_{m} R_{R}^{2}+2 R_{R} R_{s} L_{m}+L_{l} R_{R}^{2} \\
R_{s} R_{R}^{2}+\omega_{r}^{2} L_{m}^{2} R_{s}
\end{array}\right]
\end{aligned}
$$

One can see that there is redundancy in these equations and that all parameters must be positive. This can be exploited by solving this equation using only the largest subset of $\bar{\Theta}$ that gives rise to a unique and accurate estimation of $L_{l}, L_{m}, R_{s}$, and $R_{R}$. This problem is solved using standard Matlab constrained optimization tools. In the solution, a sub-set of entries of $\hat{\Theta}$ used in equation (30) is selected that has smallest estimated variance and allows for a unique solution of (30).

\section{Simulations AND Results}

\section{A. Noise Free Case}

To test the identification method and to compare its results to methods using differentiation and the indirect method, several simulations in Matlab are performed. The signals required for the identification (input: $u_{s \alpha}, i_{s \beta}$ and output: $i_{s \alpha}$ ) are generated by the state space motor model (1). This model is implemented in Simulink. The input signals of the induction motor have to be persistently exciting to identify the complete model. The transfer function (8) is difficult to identify because there is almost pole-zero cancellation in the part of the transfer function 


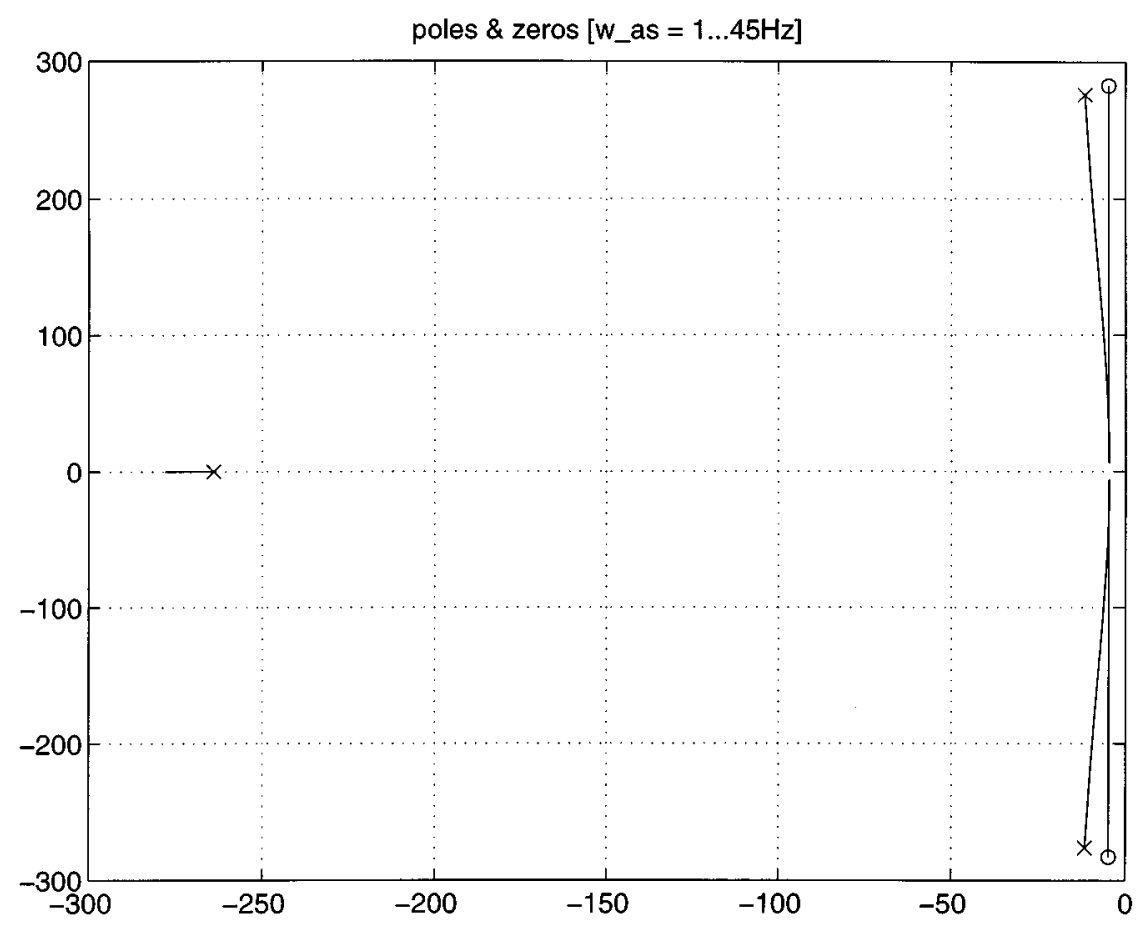

Fig. 2. Example of the root locus for input $u_{s \alpha}$, output $i_{s \alpha}$ for constant $\omega_{r}\left(\omega_{r}=1 \ldots 45 \mathrm{~Hz}\right)\left(x\right.$ and $o$ are, respectively, poles and zeros at $\left.\omega_{r}=45 \mathrm{~Hz}\right)$.
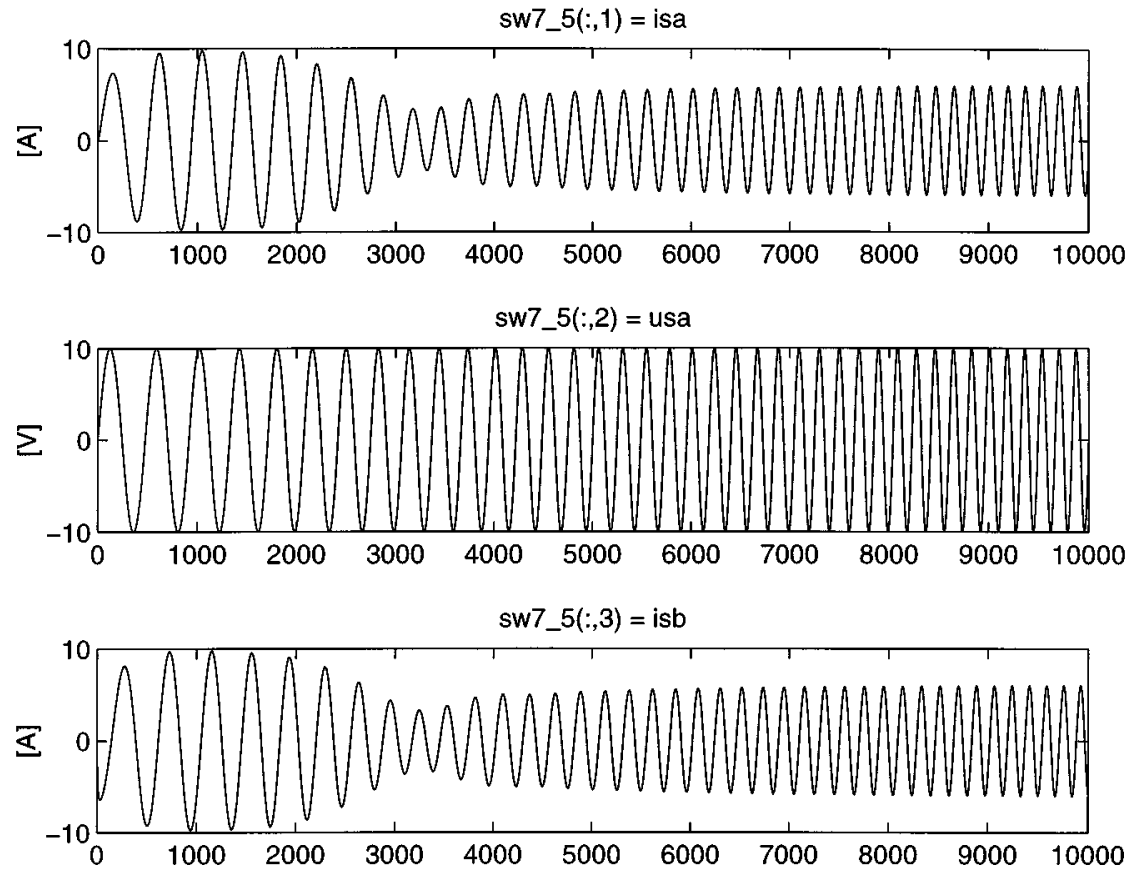

Fig. 3. Input and output signals for $\omega_{r}=2 \pi 7.5$ (horizontal axis $=$ sample number).

$i_{s \alpha} / u_{s \alpha}$. Its pole-zero plot (root locus) for different values of the constant $\omega_{r}$ is presented in Fig. 2.

The imaginary part of the zeros is at $\pm j \omega_{r}$ and the two complex poles are located very close to the zeros. To identify all poles and zeros, a signal with an high energy content around $\omega_{r}$ is needed. One method is to apply a small sweep for the stator frequency $\omega_{s}$ around $\omega_{r}$ for a constant rotor speed $\omega_{r}$. A sample frequency of $2500 \mathrm{~Hz}$ is used. An example of the resulting signals using sinewaves for low rotor speed $(\omega=2 \pi 7.5)$ is shown in Fig. 3. 


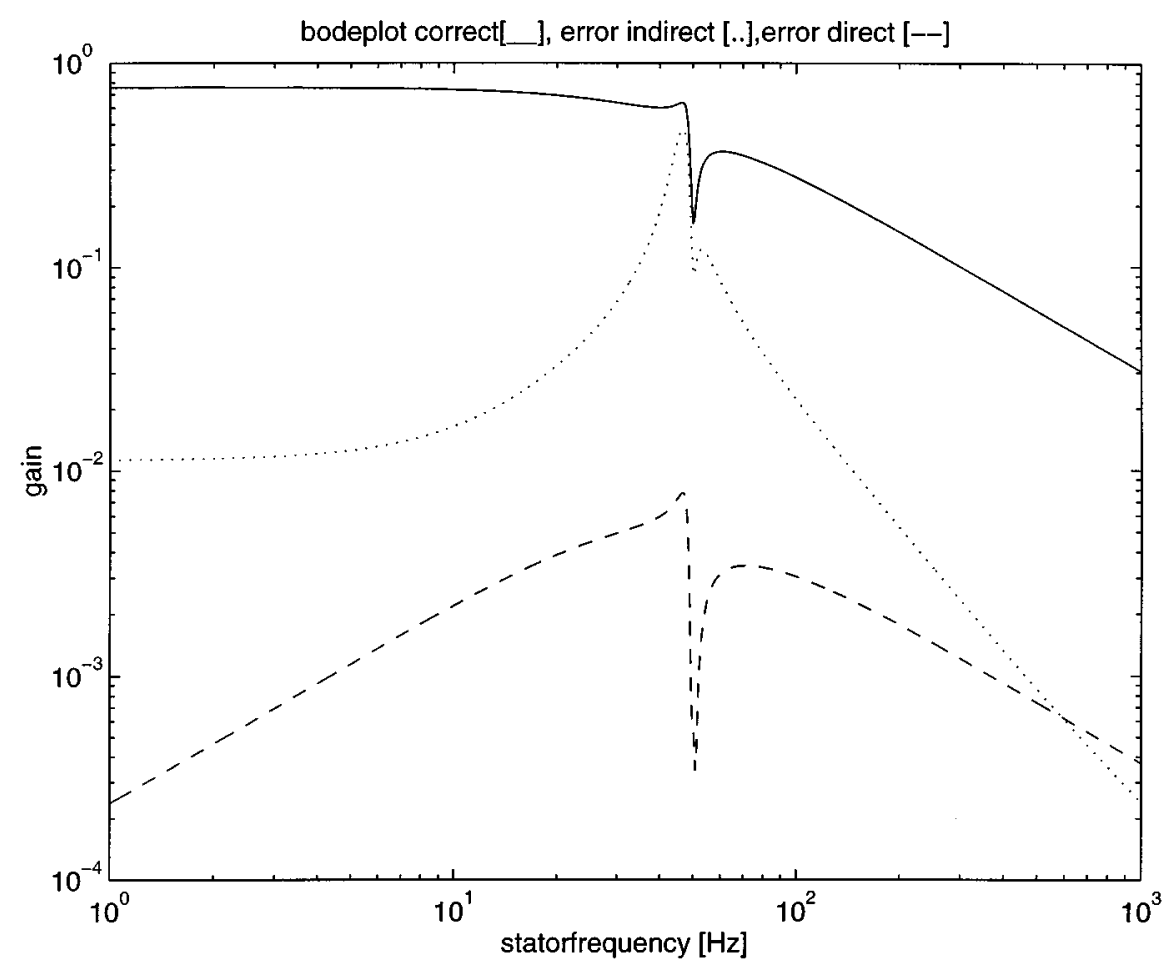

Fig. 4. Bode plot $i_{s \alpha} / u_{s \alpha}$ and identification errors—solid: correct Bode plot; dotted: error in indirect estimation; dashed: error in direct estimation.

First an attempt to identify the system applying a method of differentiation [22] is made. In this way it is not possible to identify the system.

The use of differentiation easily leads to badly conditioned least-squares problems when use is made of periodic sinusoidal signal. This is illustrated by the following.

Example 1: Let $y(t)=\sin \left(\omega_{0} t\right)$, then $\dot{y}(t)=\omega_{0} \cos \left(\omega_{0} t\right)$ and the condition number of the matrix

$$
\bar{Y}=\left[\begin{array}{cc}
y(0) & \dot{y}(0) \\
y(\Delta t) & \dot{y}(\Delta t) \\
\vdots & \vdots \\
y(N \Delta t) & \dot{y}(N \Delta t)
\end{array}\right]
$$

for $N \Delta t$ a multiplicity of the period, equals $\omega_{0}\left(\omega_{0}>1\right)$.

Another difficulty of differentiation is related to the fact that $u_{s \alpha}$ is a square wave generated by an inverter that makes differentiation not possible. Also differentiation is very sensitive to noise.

Second, an attempt is made to identify the system in the indirect way using the Matlab Identification Toolbox [16]. A discrete time system of order three is fitted to the discrete data. This model is converted to a continuous-time model using the zeroorder hold assumption $(\mathrm{ZOH})$. So we can identify the transfer function and estimate the motor parameters. The error on the estimated and true magnitude of the transfer function from $u_{s a}$ to $i_{s \alpha}$ shown in Fig. 4.

Third, we used the introduced method of identification outlined in Section III-A, extended to multiple input-single output (MISO) systems. With this method the transfer function coefficients can be identified with a small relative error (order $10^{-2}$ ) resulting in an estimated transfer function that has a small error on the magnitude plot as shown in Fig. 4. The choice of the filter parameter $\lambda$ in (15) has a very limited influence on the results of estimation of the coefficients. Once the transfer function coefficients are estimated, the motor parameters: $L_{m}$, $L_{l}, R_{s}, R_{R}$ are identified solving the constrained least-squares problem (30).

It is found that the best results are obtained when the coefficient $b_{1}$ which has the largest uncertainty in the $\Theta$ parameter vector is not used in the optimization procedure. The relative error in the approximation of the motor parameters is small (order $10^{-2}$ ). This shows that the proposed method is a good alternative for the identification of continuous-time models from sampled time data.

\section{B. Noise Added to $i_{s \alpha}$}

To get some idea on the sensitivity to noise of the new identification method, white noise is added to the output $i_{s \alpha}$. For a more general treatment of noise on data, we refer to [28]. It is found that the filter parameter $\lambda$ now has a more significant influence on the results of the identification if noise is present at the output. The best results in terms of estimated parameter variance are obtained if the pole of the filter is situated close to $s=-\omega_{r}$. Once the coefficients are found, the motor parameters are approximated using the constrained least squares optimization. The relative error in the approximation of the motor parameters is shown in Fig. 5, using different values for the filter coefficient $\lambda$. In this case $\omega_{r}$ is $2 \pi 50 \mathrm{rad} / \mathrm{s}$. In the figure the lower curve gives the relative error for the noise free case. The upper curve gives the relative error for the noisy case with $\mathrm{SNR}=37 \mathrm{~dB}$. 

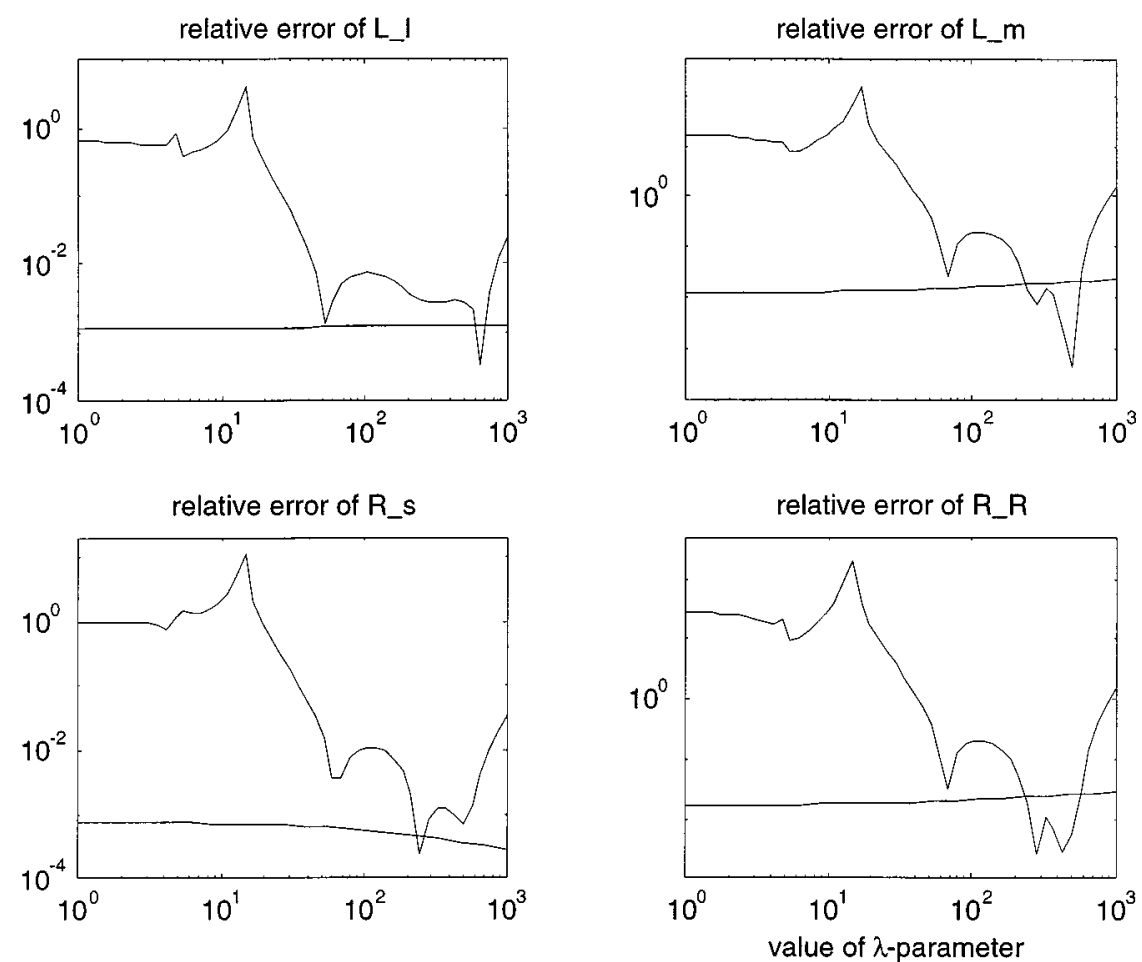

Fig. 5. Relative error in $L_{m}, L_{l}, R_{s}, R_{R}$ using different values for the filter coefficient $\lambda$ along the ordinate—upper curve: noise free; lower curve: noisy.
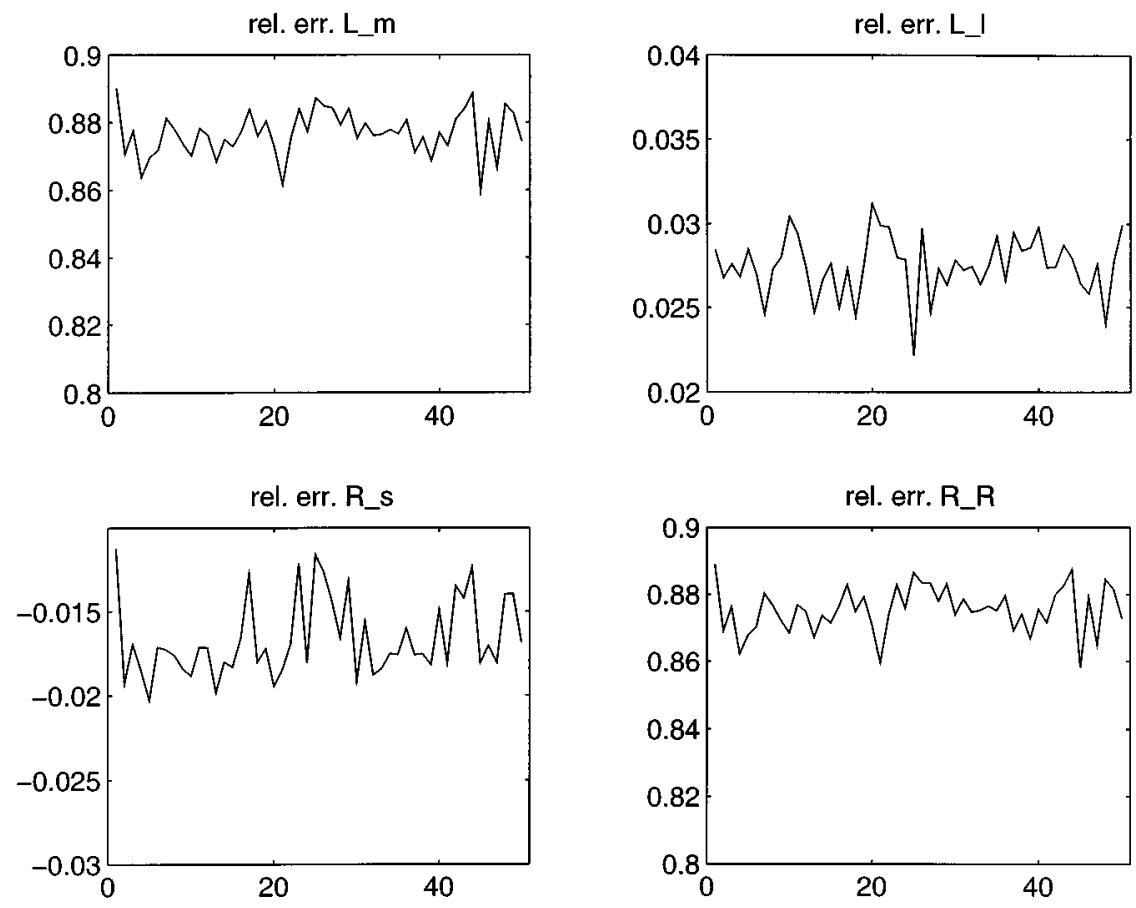

Fig. 6. Relative error in motor parameters: noisy case $\mathrm{SNR}=37 \mathrm{~dB}$.

To check whether the identification gives a robust approximation of the motor parameters a Monte Carlo experiment is done for the noisy cases with SNR: $44 \mathrm{~dB}$ and $37 \mathrm{~dB}$. The filter parameter value is taken as $\lambda=\omega_{r}$. For the noise free case the same coefficients and motor parameters are found for each ex- periment. In the experiments with $\mathrm{SNR}=44 \mathrm{~dB}$ the coefficients and parameters are estimated very well with small variation between the experiments. For the case $\mathrm{SNR}=37 \mathrm{~dB}$ the estimated parameters are shown in Fig. 6. This figure shows small variance though biased estimates. The latter is a consequence of not 


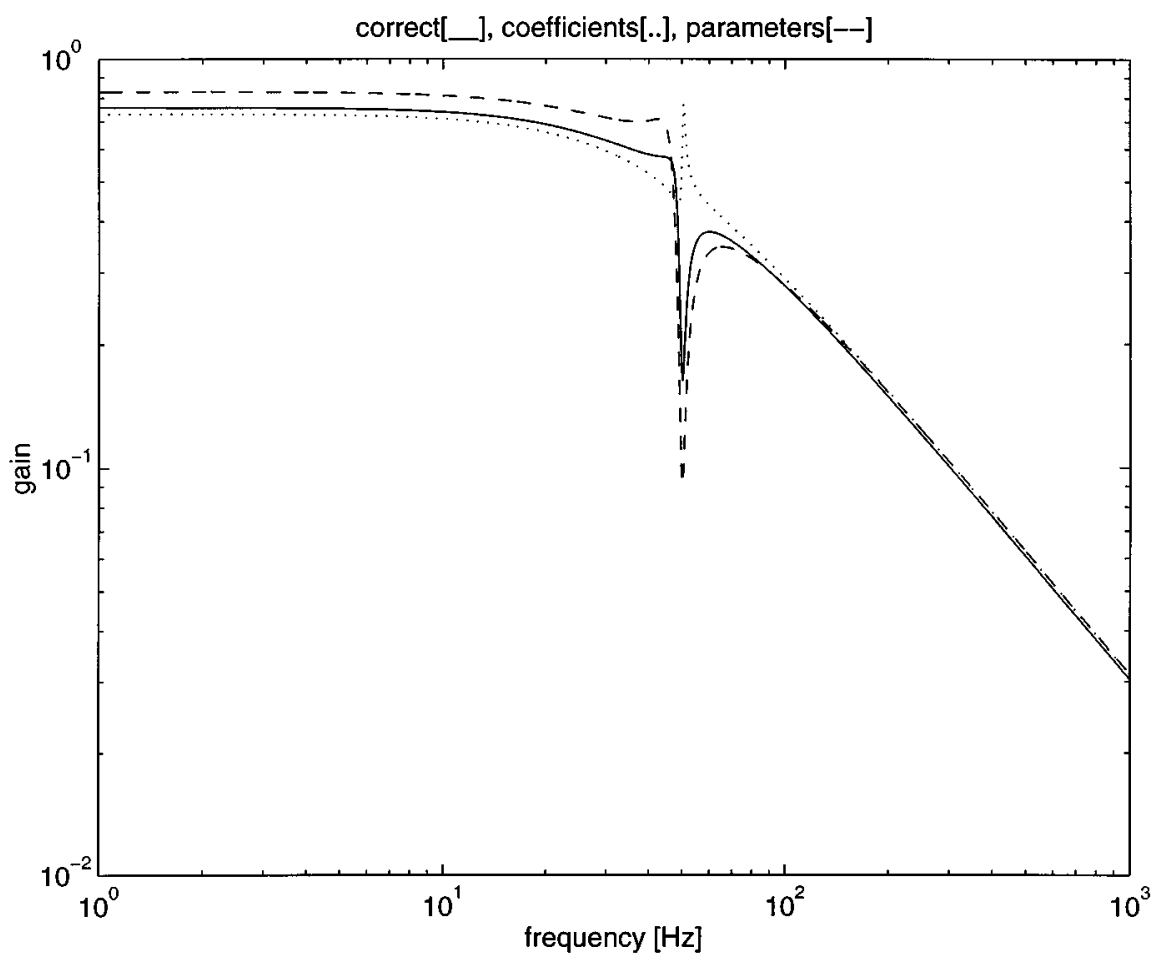

Fig. 7. Bode plots—(solid): correct transfer function; (dotted): transfer function based on estimated coefficients; (dashed): transfer function based on estimated physical parameters.

properly taken the noise into account. The goal of this paper is the outline of the basics of the method and its application to the identification of induction motors. We refer the interested reader to [28] for more details in realistic noise circumstances in the identification of continuous time systems.

Comparison of the Bode plots of the correct transfer function and the Bode plots of the estimated transfer function (i.e., after step 1) in the noisy case $\mathrm{SNR}=37 \mathrm{~dB}$ shows that the poles and zeros of the estimate are wrong. However when the motor parameters are estimated from the transfer function estimates using the redundancy in the coefficients given in (30), the poles and zeros are estimated in the correct way. This is summarized in Fig. 7. Thus, the redundancy in the equation (30) gives us a tool to get better results for the estimated motor parameters.

\section{CONCLUSION}

In this paper the problem of identifying the parameters of an induction motor from sampled data is studied. A new identification method using linear filtering instead of differentiation is introduced. The applicability of the new method over two existing approaches is explained.

The existing approach using differentiation, has two major drawbacks. First it gives rise to an ill-conditioned parameter estimation problem. Second it cannot deal with square waveforms generated by an inverter.

However, the approach presented in this paper can overcome both drawbacks. It is also shown that the new method gives better results than the indirect method. The new method gives a robust approximation of the motor parameters in noisy environments.

\section{REFERENCES}

[1] L. J. Garcés, "Parameter adaptation for the speed-controlled static AC drive with a squirrel-cage induction motor," IEEE Trans. Ind. Applicat., vol. IA-16, pp. 173-178, Mar. 1980.

[2] R. Gabriel and W. Leonhard, "Microprocessor control of induction motor," in Conf. Rec. 1982 EEE-IAS Annu. Meet., 1982, pp. 385-396.

[3] T. Irisa, S. Takata, R. Ueda, T. Sonoda, and T. Mochizuki, "A novel approach on parameter self-tuning method in AC servo system," in IFAC Contr. Power Electron. Electr. Drives, Lausanne, Switzerland, 1983, pp. $41-48$.

[4] T. Matsuo and T. A. Lipo, "A rotor parameter identification scheme for vector-controlled induction motor drives," IEEE Trans. Ind. Applicat., vol. IA-21, pp. 624-632, May 1985.

[5] C. G. Verghese, J. H. Lange, and L. F. Casey, "Analysis of instability in electical machines," IEEE Trans. Ind. Applicat., vol. 22, pp. 852-864, 1986.

[6] C. Wang, D. W. Novotny, and T. A. Lipo, "An automated rotor time constant measurement system for indirect field-oriented drives," IEEE Trans. Ind. Applicat., vol. 24, pp. 151-159, Jan. 1988.

[7] H. Schierling, "Fast and reliable commissioning of AC variable speed drives by self-commissioning," in Conf. Rec. 1988 IEEE-IAS Annu. Meet., 1988, pp. 489-492.

[8] R. D. Lorenz and D. B. Lawson, "A simplified Approach to continuous, on-line tuning of field oriented induction machine drives," in Conf. Rec. 1988 IEEE-IAS Annu. Meet., 1988, pp. 444-449.

[9] J. R. Willis, G. J. Brock, and J. S. Edmonds, "Derivation of induction motor models from standstill frequency response tests," IEEE Trans. Energy Conv., vol. 4, pp. 608-613, Dec. 1989.

[10] M. Depenbrock and N. R. Klaes, "Determination of the induction machine parameters and their dependencies on saturation," in Conf. Rec. IEEE-IAS Аnпи. Meet., 1989, pp. 17-22.

[11] S. K. Sul, "A novel technique of rotor resistance estimation considering variation of mutual inductance," IEEE Trans. Ind. Applicat., vol. 25, pp. 578-587, July 1989.

[12] M. Vélez-Reyes, K. Minami, and G. C. Verghese, "Recursive speed and parameter estimation for induction machines," in Conf. Rec. 1989 IEEE-IAS Апnи. Meet., 1989, pp. 607-611.

[13] T. Saitoh, T. Okuyama, and T. Matsui, "An Automated secondary resistance identification scheme in vector controlled induction motor drives," in Conf. Rec. 1989 IEEE-IAS Annu. Meet., 1989, pp. 594-600. 
[14] K. T. Hung and R. D. Lorenz, "A rotor flux error-based, adaptive tuning approach for feedforward field oriented induction machine drives," in Conf. Rec. 1990 IEEE-IAS Annu. Meet., 1990, pp. 589-594.

[15] S. Van Huffel and H. Zha, "The restricted total least squares problem: Formulation, algorithm and properties," SIAM J. Matrix Anal. Applicat., pp. 292-309, 1991.

[16] L. Ljung, System Identification Toolbox for use with Matlab. Natick, MA: The MathWorks. Inc., July 1991

[17] A. M. Khambadkone and J. Holtz, "Vector-controlled induction motor drive with a self-commissioning scheme," IEEE Trans. Ind. Electron., vol. 38, pp. 322-327, Oct. 1991.

[18] J. Holtz and T. Thimm, "Identification of the machine parameters in a vector-controlled induction motor drive," IEEE Trans. Ind. Applicat., vol. 27, pp. 1111-1118, Nov. 1991

[19] D. J. Atkinson, P. P. Acarnley, and J. W. Finch, "Observers for induction motor state and parameter estimation," IEEE Trans. Ind. Applicat., vol. 27, pp. 1119-1127, Nov. 1991

[20] J. C. Moreira, K. T. Hung, T. A. Lipo, and R. D. Lorenz, "A simple and robust adaptive controller for detuning correction in field-oriented induction machines," IEEE Trans. Ind. Applicat., vol. 28, pp. 1359-1366, Nov. 1992.

[21] A. Tzrynadlowski, The Field Orientation Principle in the Control of Induction Motor Drives. Boston, MA: Kluwer, 1994.

[22] C. Moons and B. de Moor, "Parameter identification of induction motor drives," Automatica, vol. 31, no. 8, pp. 1137-1147, 1995.

[23] S. Bigi, T. Söderström, and B. Carlsson, "An IV-schema for estimating continuous-time stochastic models from discrete-time data," in Proc. 10th Int. IFAC Symp. Syst. Identification, Copenhagen, Denmark, 1994, pp. $645-650$.

[24] S. I. Moon and A. Keyhani, "Estimation of induction machine parameters from standstill time-domain data," IEEE Trans. Ind. Applicat., vol. 30, pp. 1609-1615, Nov. 1994

[25] J. Stephan, M. Bodson, and J. Chiasson, "Real-time estimation of the parameters and fluxes of induction motors," IEEE Trans. Ind. Applicat., vol. 30, pp. 746-757, May 1994.

[26] H. Borsting, M. Knudson, H. Rasmussen, and P. Vadstrup, "Estimation of physical parameters in induction motors," SYSID'94, vol. 2, pp. 553-558, 1994

[27] D. E. Borgard, G. Olsson, and R. D. Lorenz, "Accuracy issues for parameter estimation of field oriented induction machine drives," IEEE Trans. Ind. Applicat., vol. 31, no. 4, pp. 795-801, July. 1995.

[28] C. T. Chou and M. Verhaegen, "Continuous time identification of SISO systems using laguerre functions," in 11th IFAC Symp. Syst. Identification, vol. 3, pp. 1349-1353.

[29] S. Wade, M. W. Dunnigan, and B. W. Williams, "A new method of rotor resistance estimation for vector-controlled induction machines," IEEE Trans. Ind. Electron., vol. 44, pp. 247-257, Mar. 1997.

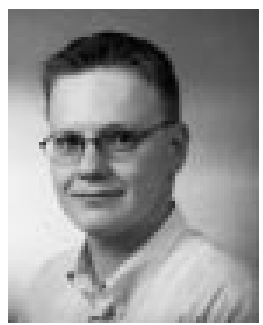

Richard F. F. Koning was born in Den Helder, The Netherlands, in 1971. He received the B.S. and M.S. degrees in electrical engineering from the Delft University of Technology, Delft, The Netherlands, in 1996. His graduation project was concerning the modeling and control of asynchronous machines.

$\mathrm{He}$ is currently working as a Sales Manager at the former NKF Cable B.V. Delft in the Netherlands (Nowadays Pirelli Cables \& Systems N.V.). After graduation he started at NKF Cable as a Product Development Engineer in the high-voltage research laboratory and did research and development on intelligent cable systems with integrated optical fibers. As Manager Development Engineering at NKF Services he did research and development in onsite diagnostics and testing of power cable systems. He published papers about this subject in cooperation with the Delft University of Technology, Dutch electricity utilities and manufacturers of high-voltage test equipment.

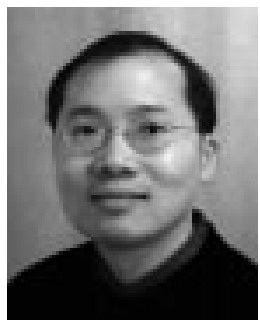

Chun Tung Chou was born in Hong Kong in 1967. $\mathrm{He}$ received the B.A. degree in engineering science from the University of Oxford, Oxford, U.K., in 1988 and the $\mathrm{Ph} . \mathrm{D}$. degree in control engineering from the University of Cambridge, Cambridge, U.K., in 1994.

$\mathrm{He}$ is currently a Postdoctoral Research Fellow at the Electrical Engineering Department of the Delft University of Technology, Delft, The Netherlands. His current research interests are system identification of linear and nonlinear systems.

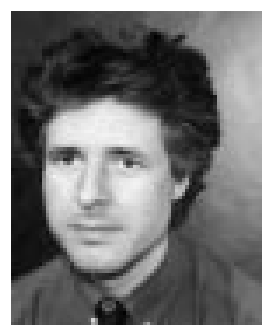

Michel H. G. Verhaegen received the bachelor's degree in engineering/aeronautics from the Delft University of Technology, Delft, The Netherlands, in August 1982, and the Ph.D. degree in applied sciences from the Catholic University, Leuven, Belgium, in November 1985.

During his graduate study, he held an IWONL research assistantship in the Department of Electrical Engineering. From 1985 to 1987, he was a Postdoctoral Associate of the U.S. National Research Council and during that time was affiliated with the NASA Ames Research Center in California. From 1988 to 1989, he was an Assistant Professor at the Delft University of Technology, Department of Electrical Engineering. Since February 1990, he has been a Senior Research Fellow of the Dutch Academy of Arts and Sciences and affiliated with the Network Theory Group of the Delft University of Technology. Since 1990, he has held short sabbatical leaves at the University of Uppsala, Sweden, McGill University, Montreal, PQ, Canada, the University of Lund, Lund, Sweden, and the German Aerospace Research Center (DLR), Munich. From 1994 to 1999, he was an Associate Professor of the control laboratory of the Delft University of Technology. Currently he is full Professor of the faculty of Applied Physics of the University of Twente, Enschede, The Netherlands, where he is heading the Systems and Control Engineering Group. His main research interest is the interdisciplinary domain of numerical algebra and system theory. In this field he has published more than 100 papers. Current activities focus on the development and practical application of new identification and simulation tools in model-based industrial controller design.

Prof. Verhaegen received a Best Presentation Award at the American Control Conference, Seattle, WA, 1986 and a Recognition Award from NASA in 1989. $\mathrm{He}$ is project leader of a number of research projects sponsored by the European Community, the Dutch National Science Foundation (STW) and the Dutch industry.

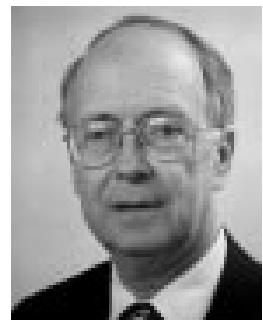

J. Ben Klaassens was born in Assen, The Netherlands, in 1942. He received the B.S., M.S., and Ph.D. degrees in electrical engineering from the Delft University of Technology, Delft, The Netherlands.

$\mathrm{He}$ is currently Associate Professor at the Control Laboratory of the Delft University of Technology. His work has been concerned with inverter circuits. pulse width modulation and the control of electrical machinery. His research work and professional publications are in the area of converter systems with high internal pulse frequencies for submegawatt power levels employing thyristors, power transistors and IGBTs. His interests include modeling and control of converters and electrical drives. Dr. Klaassens has published a variety of papers on series-resonant converters for low and high power applications. He has designed and built prototypes of the early $\mathrm{dc}-\mathrm{dc}$ to ac-ac series-resonant converters for a wide variety of applications such as electric motors and generators, communication power supplies, radar signal generators, arc welders and space applications. His current research project is a part of the national first, all modes, all sizes (FAMAS) Programme. This program includes the modeling and the control of the dynamic behavior of the so-called Jumbo Container Crane and the computation of the time-optimal trajectories for transporting the container.

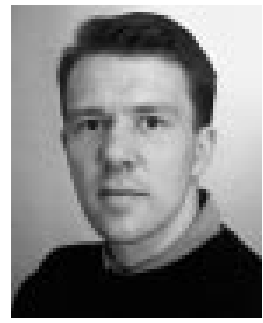

Jeroen R. Uittenbogaart was born in Rotterdam, The Netherlands, in 1967. He received the M.Sc. degree in mechanical engineering from the Eindhoven University of Technology, Eindhoven, The Netherlands.

$\mathrm{He}$ is currently Project Engineer at Vekoma Manufacturing in Vlodrop, The Netherlands. His work has been concerned with process control and plant-wide optimization, mechatronics and the control of electrical machinery. His current interest is in the design of rollercoaster launch systems based on linear synchronous motors. 